\title{
Prácticas de bioseguridad en lecherías comerciales de la Zona Central de Chile
}

Biosecurity practices in commercial dairy farms in Central Chile

\author{
Carla Velásquez P., MV, Mario Duchens A., MV, MS, PhD \\ Universidad de Chile, Facultad de Ciencias Veterinarias y Pecuarias. Casilla 2, Santiago 15, \\ Chile
}

\section{ABSTRACT}

Biosecurity is defined as the set of measures designed to reduce the entry of pathogens to the herd as well as to avoid or reduce their dissemination within the herd. Biosecurity status is becoming increasingly important, since it influences herd health and product quality. However, biosecurity status in dairy farms in Chile is not known.

The aim of the present study was to describe the main biosecurity practices carried out in commercial dairies of the Central Zone of Chile. To carry out this, a survey was designed and subsequently applied to 33 dairy farms located in the regions of Valparaiso, O'Higgins and Metropolitan. This activity was complemented with a visit and inspection of the dairy.

Regarding to the arrival of cattle from other farms, in 13 dairies (39.4 \%) new cattle were brought into the herd during the last 2 years. Heifers were the class most frequently brought. In 6 of these dairies (46.2\%), quarantine to the incoming animals was carried out. In relation to the people's traffic, in 8 operations (24.2\%) there was some kind of signposting in the entry of the facilities and only in one (3\%), visitors had to clean and disinfect its footwear before entering to the facilities. Regarding vehicle traffic, only two operations (6\%) keep a process of disinfection for the wheels of the vehicles entering to the facilities.

In all the dairies surveyed there were other animal species, which, in the majority of the cases, had direct contact with the bovines or with its sources of water or food. The species observed with the highest frequencies were: cats $(90.9 \%)$, dogs $(72.7 \%)$, horses $(60.6 \%)$, and poultry (36.4\%). In relation to the food management, in 17 cases $(51.5 \%)$ the remaining food of the adult cattle was used in the ration of another group of females, being the most frequent destination the rations of dry cows and weaned calves.

It is concluded that in general the biosecurity status of the dairies surveyed is insufficient. The most deficient areas regarding biosecurity practices are people's entrance and control, and the contact of cattle with other domestic species. However, there is a low degree of inclusion of foreign animals into the herd, since it is widely acknowledged that keeping a closed herd is an effective way to prevent the access of pathogens to the farm.

Keywords: Biosecurity, practices, dairies, cattle, herd. 


\section{RESUMEN}

La bioseguridad se define como el conjunto de medidas tendientes a reducir la entrada de patógenos a la explotación animal así como a evitar o disminuir su diseminación dentro del predio. Hoy en día, la situación de bioseguridad adquiere gran importancia, ya que influye en la salud del rebaño y la calidad del producto final.

El presente estudio tuvo por objetivo describir las principales prácticas de bioseguridad que se aplican en lecherías comerciales de la Zona Central de Chile. Para esto, se elaboró una encuesta que se realizó en 33 predios de las regiones de Valparaiso, O’Higgins y Metropolitana. Esta actividad se complementó con una visita e inspección del plantel.

En relación al ingreso de bovinos desde otros predios, en 13 lecherías $(39,4 \%)$ se incorporó algún tipo de ganado nuevo a su rebaño, durante los últimos 2 años. El tipo de ganado más frecuente de ingresar fueron las vaquillas. De estas lecherías, en 6 de ellas (46,2\%) se realizó cuarentena de los animales ingresados. En relación al ingreso de personas, en 8 predios $(24,2 \%)$ había algún tipo de señalización en la entrada del predio y sólo en una (3\%) los visitantes debían limpiar y desinfectar su calzado antes de ingresar a las instalaciones. Para el ingreso de vehículos, sólo dos planteles (6\%) mantenían un rodiluvio o proceso de desinfección para las ruedas de los vehículos que ingresaban al plantel.

En todas las lecherías encuestadas existían otras especies de animales, donde la mayoría de ellas, tenían contacto directo con los bovinos o con sus fuentes de agua o de alimento. Las especies observadas con mayor frecuencia fueron: gatos $(90,9 \%)$, perros $(72,7 \%)$, caballos $(60,6 \%)$ y aves de corral (36,4\%). Respecto al manejo alimentario, en 17 casos (51,5\%), el alimento sobrante de los animales adultos era utilizado en la ración de otro grupo de hembras, siendo su destino más frecuente la ración de las vacas secas y las terneras en recría.

Se concluye que en general la situación de bioseguridad en las lecherías encuestadas es insuficiente. Las áreas más deficientes en prácticas de bioseguridad fueron el ingreso y control de personas externas al predio y el contacto de los bovinos con otras especies domésticas. Sin embargo, existe una baja incorporación de animales desde otros predios, ya que se reconoce que mantener un rebaño cerrado es una medida eficaz para prevenir el ingreso de patógenos al plantel.

Palabras claves: Bioseguridad, prácticas, lecherías, bovinos, rebaño. 


\section{INTRODUCCIÓN}

Las medidas de bioseguridad se han utilizado en medicina veterinaria desde hace muchos años, como parte de los programas de erradicación y control de enfermedades. Actualmente, eventos como los brotes de fiebre aftosa y encefalopatía espongiforme bovina, la globalización del comercio agrícola y la creciente preocupación de los consumidores por adquirir alimentos sanos y seguros, han hecho que la bioseguridad adquiera una gran relevancia tanto a nivel nacional como a nivel de planteles individuales.

La bioseguridad a nivel de un plantel lechero comprende la implementación de prácticas de manejo destinadas a prevenir la introducción de agentes causantes de enfermedades dentro de la operación, los cuales pueden ser infecciosos o no infecciosos, por ejemplo tóxicos (USDA, 2004; Wells, 2000; Barefoot, 2002; Dargatz et al, 2002). Adicionalmente, las medidas diseñadas e implementadas para prevenir la diseminación de patógenos o agentes tóxicos entre los distintos grupos de animales de un rebaño, recibe el nombre de biocontención (Dargatz et al, 2002; USDA, 2004; Radostits, 2003). Ambas estrategias son indispensables para minimizar los impactos potenciales que una enfermedad puede provocar en un plantel lechero comercial (Dargatz et al., 2002; Wells, 2002; Wolfgang, 2002, USDA, 2004).

Los componentes de la bioseguridad incluyen, principalmente, programas de introducción de nuevos animales dentro del predio, incluyendo el conocimiento de su fuente de origen e historial sanitario, aislamiento de los nuevos animales y realización de pruebas diagnósticas para las enfermedades más importantes del ganado bovino. También se incluyen programas de vacunación estratégicos, prácticas de higienización, que incluyen los procedimientos de ordeña, desinfección del equipo y manejo del estiércol, control de visitas y vehículos, técnicas correctas para el manejo de animales sanos y enfermos, control del tránsito animal, procedimientos de limpieza de ropas y materiales de trabajo, y control de la exposición del rebaño a otras especies de animales, insectos y plagas (USDA, 2004; Dargatz et al, 2002).

Un programa de bioseguridad efectivo, sin embargo, necesita ser más que una lista genérica de cosas a realizar; debe ser un foco de decisión flexible y adaptarse a las situaciones particulares de cada plantel lechero, ya que cada plantel tiene sus propias ventanas de vulnerabilidad y cada productor posee diferentes preocupaciones y percepciones del riesgo (Dargatz et al, 2002; Wells, 2002).

En las industrias avícola y porcina, las prácticas de bioseguridad están incorporadas en los procedimientos rutinarios de la producción. La producción de leche de calidad requiere también de la implementación de prácticas de bioseguridad y biocontención eficientes. Sin embargo, en la producción bovina de leche en general no se conoce a cabalidad la situación de bioseguridad predial y varios estudios en otros países muestran que la mayoría de los productores no aplica prácticas básicas de bioseguridad.

En Chile, se desconoce el grado de conocimiento y adopción de medidas de bioseguridad en lecherías, pero posiblemente en la mayoría de los predios hay una situación similar a la 
descrita anteriormente. Adicionalmente, los rebaños de la Zona Central se han reducido en cantidad pero han aumentado en tamaño y complejidad. La expansión en muchos casos ha provenido de la adquisición de animales de otros predios, lo que sumado al prolongado confinamiento de los animales; el ingreso de insumos externos y la acumulación de residuos, hace que estos sistemas sean propensos a sufrir pérdidas devastadoras debido a epidemias de enfermedades infecciosas. Esto hace que la implementación y evaluación de planes de bioseguridad a nivel predial, sea cada vez una tarea de mayor importancia.

Mediante este estudio se pretende describir las prácticas de bioseguridad que son aplicadas por los productores lecheros en la Zona Central de nuestro país.

\section{MATERIAL Y MÉTODOS}

El estudio sobre prácticas de bioseguridad se realizó a través de una encuesta, la cual se aplicó en 33 lecherías comerciales ubicadas en la Zona Central de Chile, abarcando las regiones de Valparaíso (10 predios), Metropolitana (12 predios) y de O'Higgins (11 predios). Como requisito, los planteles debían tener un mínimo de 80 vacas en ordeña para ser evaluados.

La encuesta se elaboró a partir de los manuales de bioseguridad en producción de leche elaborados por la autoridad competente de países como EEUU, Canadá, Australia, Nueva Zelanda, Inglaterra, Irlanda, Argentina y Uruguay. Se consultó además con expertos de Facultades de Veterinaria de Chile y Estados Unidos y profesionales del Servicio Agrícola y Ganadero. Finalmente se elaboró un cuestionario constituido por 122 preguntas, basadas en la caracterización y cumplimiento de las medidas tendientes a evitar el ingreso de patógenos al predio, prevenir la diseminación de los patógenos dentro del predio y controlar su egreso hacia predios vecinos. La mayoría de las preguntas eran cerradas y tuvieron sólo tres alternativas de respuesta: "SI"; "NO"; y "NO APLICA", el resto de las preguntas poseen respuestas numéricas o de alternativas. Para organizar con mayor claridad su análisis, la encuesta se dividió en tres secciones:

1. Información básica del predio

2. Adopción de medidas de bioseguridad generales

3. Medidas específicas según enfermedad, donde se realizan preguntas especificas en relación al manejo de las enfermedades más importantes del rebaño lechero, como son la tuberculosis, brucelosis bovina, mastitis contagiosa, leucosis, paratuberculosis, diarrea viral bovina y neosporosis. 
La encuesta fue realizada en el predio y al mismo tiempo, se llevó a cabo una inspección y evaluación visual del plantel, sus animales e infraestructura, con el fin de corroborar personalmente el cumplimiento de las medidas de bioseguridad de acuerdo a lo contestado.

\section{Análisis de la información:}

El análisis de la información recolectada es descriptivo, presentándose los resultados como frecuencias absolutas y porcentajes de cumplimiento.

Para poder evaluar los predios según tamaño del rebaño, se consideró el número de vacas masa (vacas en lactancia + vacas secas) de todas las lecherías encuestadas, el cual fue dividido en cuartiles y categorizado en 4 ítems para su posterior análisis. Las categorías utilizadas fueron: plantel pequeño, intermedio, grande y muy grande, esto se realizó para evaluar alguna diferencia entre los distintos predios para la adopción de prácticas de bioseguridad.

\section{RESULTADOS Y DISCUSIÓN}

A continuación, se muestran los resultados más relevantes relacionados con las prácticas de bioseguridad implementadas en lecherías de la Zona Central de Chile:

\section{Ingreso de animales:}

Durante el estudio, 13 predios $(39,40 \%)$ del total de planteles encuestados incorporaron algún tipo de ganado, durante los últimos 2 años. Siendo el ingreso de nuevos animales a un plantel lechero, uno de los mayores riesgos para la bioseguridad (Wells, 2000; Radostits, 2001; USDA, 2004), ya que mediante esta vía es posible que sean introducidos nuevos agentes patógenos al plantel o vuelvan a reintroducirse agentes que ya estaban erradicados. Lo ideal es tener un rebaño completamente cerrado, pero muchas veces, debido a la necesidad de aumentar el número de animales o por las altas tasas de eliminación, los reemplazos no son suficientes y los planteles incorporan animales desde otros predios. Según el estudio la clase de ganado más frecuente de ingresar fueron las vaquillas, seguida por las vacas en lactancia y en menor grado las terneras y toros.

De las lecherías que ingresaron bovinos a su plantel, 6 de ellas (46,20\%) realizaron cuarentena de los animales nuevos, concepto que no está incorporado en los sistemas de manejo y que corresponde a un punto de riesgo sanitario para los animales propios.

\section{Ingreso de personas:}

Los visitantes o empleados pueden también incorporar agentes patógenos al plantel, participando principalmente como vectores mecánicos. Es por ello, que dentro de las medidas de bioseguridad se considera el control y registro de todas las visitas que ingresan a un plantel lechero, siendo de suma importancia aquellas personas que trabajan 
directamente con animales o que visitan distintos predios durante una jornada (Hoe y Ruegg, 2006). De acuerdo a los antecedentes recolectados en lecherías de la Zona Central, 8 planteles $(24,2 \%)$ poseían algún tipo de señalización en la entrada del predio. En 7 lecherías $(21,20 \%)$ existían restricciones para el ingreso de personas ajenas al plantel, incluyendo invitados, técnicos de inseminación artificial, veterinarios o vendedores. Sólo en una lechería (3\%) los visitantes limpiaban y desinfectaban sus botas o zapatos antes de ingresar a las instalaciones.

Durante las visitas realizadas a los predios, es relevante mencionar que en la mayoría de ellos, no existía algún tipo de señalización o advertencia que guiara a los visitantes, siendo el acceso a las instalaciones y corrales de los animales, totalmente libre.

\section{Existencia de otras especies de animales en el plantel lechero:}

En todas las lecherías encuestadas (100\%) existían otras especies de animales en el plantel, donde la mayoría de ellas, tenían contacto directo con los bovinos o con sus fuentes de agua o de alimento (Ver foto 1). Las especies que fueron observadas con mayor frecuencia en las lecherías fueron: gatos, perros, caballos y aves de corral (principalmente gallinas) (Tabla 1)

Tabla 1: Existencia de otras especies de animales en planteles lecheros de la Zona Central

\begin{tabular}{||c|c|c||}
\hline Especie animal & $\begin{array}{c}\text { Número de planteles } \\
\text { en que se observaron }\end{array}$ & $\begin{array}{c}\text { Porcentaje de planteles } \\
\text { en que se observaron }\end{array}$ \\
\hline Gatos & 30 & 90,9 \\
\hline Perros & 24 & 72,7 \\
\hline Equinos & 20 & 60,6 \\
\hline Aves de corral & 12 & 36,4 \\
\hline Ganado para carne & 11 & 33,3 \\
\hline Camélidos & 2 & 6 \\
\hline Cerdos & 1 & 3 \\
\hline Ovejas & 0 & 0 \\
\hline Cabras & 0 & 0 \\
\hline
\end{tabular}

La presencia de otras especies de animales en un plantel, puede ser un factor de riesgo para la bioseguridad, ya que pueden ser fuente de una variedad de enfermedades infecciosas (Radostits, 2001). La diseminación de agentes patógenos puede ocurrir por el contacto físico directo con el ganado de cualquier edad o mediante el contacto indirecto con su comida o fuentes de agua. Por ejemplo; los perros son el hospedero intermediario y definitivo de $N$. caninum (McAllister et al., 1998; Lindsay et al., 1999; Basso et al., 2001; Dubey et al., 2002), las aves silvestres son portadoras de Salmonella y los pequeños rumiantes al igual que los cerdos pueden padecer de fiebre aftosa y diseminarla al resto del rebaño. 


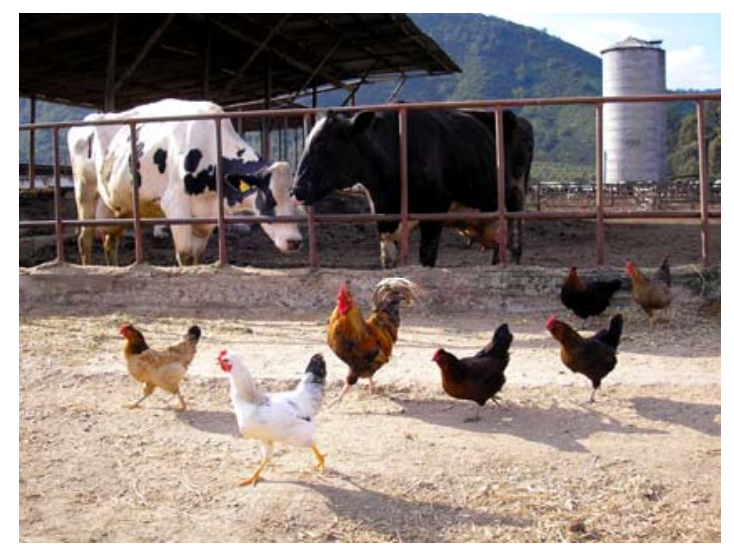

Foto 1: Presencia de otras especies de animales (aves domésticas) cerca de las instalaciones para los bovinos (comederos y bebederos), detectado en varias lecherías de la Zona Central bajo estudio

\section{Manejos del ternero recién nacido}

De las lecherías estudiadas, sólo en 3 de ellas (9\%) se constató que el recién nacido no tenía contacto con otros animales adultos después del parto, ya que en estos casos los partos ocurrían en maternidades individuales y la permanencia del ternero con su madre era la mínima posible. Sin embargo, en la mayoría de las lecherías los partos ocurrían en corrales colectivos o en un corral de preparto (en 29 planteles), donde el ternero tenía contacto con otros animales adultos y con terneros neonatos de otras hembras después de nacer (Ver foto 2). Este manejo podría representar un riesgo para la salud de los terneros recién nacidos, ya que su permanencia en este corral les permite el contacto con fecas de ganado adulto y otras fuentes potenciales de patógenos.

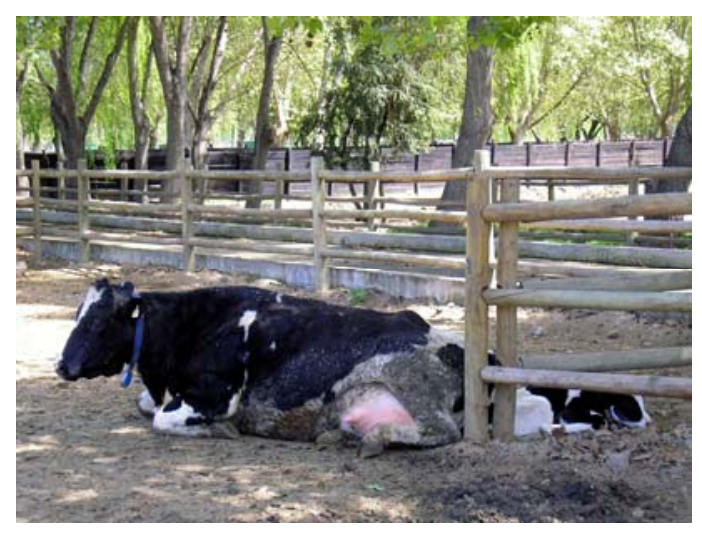

Foto 2: En la mayoría de los planteles lecheros, las hembras paren en corrales colectivos, donde el ternero tiene contacto con otros bovinos.

En relación al tiempo de permanencia del ternero con su madre, el promedio de horas entre las distintas lecherías estudiadas fue de 29,8. Sin embargo, los rangos de horas en que permanecía el ternero recién nacido con su madre era muy variado entre los distintos planteles lecheros, con un mínimo de 0 hrs. (en 3 predios) hasta un máximo de 7 días (en un plantel). De los planteles lecheros encuestados, sólo 3 retiraban inmediatamente al ternero 
recién nacido de su madre y no le permitían su asistencia, ya que reconocían que ésta medida era fundamental para prevenir el traspaso de patógenos desde la madre al ternero.

Según los datos recolectados en el estudio, en 5 planteles lecheros administraban a sus terneras recién nacidas leche de descarte como fuente de alimentación, en 3 predios les suministraban leche pasteurizada y en la mayoría de los planteles, eran alimentadas con sustituto lácteo durante su lactancia (recomendación para controlar la diseminación de agentes infecciosos dentro del rebaño). La práctica de alimentar a las terneras con leche de descarte, es un grave problema para la bioseguridad de un rebaño, ya que puede conducir a un incremento de la morbilidad y mortalidad de los terneros debido a la ingestión de agentes patógenos (Stabel et al, 2004).

\section{Manejo entre animales jóvenes y adultos:}

En lecherías de la Zona Central, es común que el alimento sobrante de los comederos de las vacas en producción (denominado reciclado), sea administrado a otro grupo de animales. Se pudo constatar que en 17 planteles lecheros (51,5\%), el alimento sobrante de los animales adultos (reciclado) era utilizado en la ración de otro grupo de hembras bovinas. De este total, el destino más frecuente del reciclado correspondió a la ración de grupos de menores requerimientos; como crianza y vacas secas. Esta práctica constituye un peligro para la bioseguridad, ya que mediante esta vía es posible que patógenos de los animales adultos sean transmitidos a otros grupos de animales o a los más jóvenes. Al mismo tiempo, el alimento se puede deteriorar y contaminar (por ejemplo con hongos), representando un riesgo para los bovinos.

\section{Prácticas de vacunación:}

De los datos analizados, el $100 \%$ de las lecherías aplicaba algún tipo de vacuna a su rebaño. Sin embargo, 22 lecherías poseían un plan de vacunación escrito y elaborado por su propio veterinario a cargo. En promedio, según los datos recolectados, cada plantel lechero vacunaba rutinariamente contra 7 enfermedades a su rebaño.

Es importante recordar que una correcta práctica de vacunación puede reducir la incidencia de una enfermedad en particular, disminuir la gravedad de ciertos cuadros clínicos, mejorar la respuesta inmune de los bovinos, pero no proveer una inmunidad completa para el rebaño, ya que se deben implementar otras medidas para prevenir y controlar la diseminación de enfermedades. 


\section{Uso de material desechable}

De las lecherías estudiadas, en $23(69,7 \%)$ se utilizaba sólo una vez las agujas y luego las desechaban, por lo tanto 10 predios reutilizaban las agujas en sus bovinos. En cuanto al uso de las mangas para palpación, en 23 planteles $(69,7 \%)$ se usaba sólo una vez la manga para palpación rectal y luego era desechada, mientras que 10 productores comentaron que las mangas para palpación rectal eran utilizadas en más de un bovino.

Este tipo de manejo, se asociaría generalmente a una falta de conocimiento (por parte del personal a cargo) del potencial peligro de transmisión de agentes infecciosos que representa y por la asociación a mayores gastos económicos en el uso de agujas y mangas para palpación desechables. Estos antecedentes mostrarían una gran falencia en la capacitación del personal, con respecto al buen manejo del material de enfermería. El uso de mangas para palpación vía rectal en más de un animal, sería responsabilidad directa del veterinario a cargo del plantel lechero, quien es el encargado de realizar el examen de diagnóstico mediante esta vía.

\section{Manejo de animales enfermos}

De los predios estudiados, sólo $13(39,4 \%)$ poseían un área especial para el cuidado de sus animales enfermos. Aparte de la baja difusión de esta medida, se observó que estas instalaciones no eran las adecuadas, ya que se ubicaban muy cerca del resto de los bovinos.

\section{Manejo de animales muertos}

De un total de 31 lecherías con información disponible, 17 de ellas (54,8\%) eliminaban adecuadamente a sus bovinos muertos, es decir, entierran a sus animales en fosas profundas selladas o queman los cadáveres de manera correcta. Sin embargo, 14 predios $(45,16 \%)$ eliminaban a sus cadáveres de forma inadecuada, siendo lo más frecuente el uso de una fosa común abierta en el predio (descrita en 7 lecherías).

La eliminación de los animales muertos en lecherías de la Zona Central es un tema que requiere planificación y regulación, ya que casi la mitad de los predios evaluados elimina inadecuadamente los cadáveres, y el resto de los planteles catalogados como adecuados no poseen un manejo estricto en este tema.

\section{Prácticas sobre el personal de lecherías}

En relación al personal encargado de los terneros, se describió que en 19 planteles ésta persona se dedica sólo a este grupo de animales, sin tener acceso a los animales adultos. Sin embargo, 14 ternereros poseen otras funciones en la lechería y tienen contacto con los 
bovinos adultos, lo que podría representar un riesgo para la bioseguridad de los bovinos más jóvenes.

Según la encuesta, 22 lecherías (66,7\%) poseían un lugar especial para la limpieza de manos y botas de los trabajadores. Sin embargo, 10 predios no poseían un lugar específico, siendo la sala de ordeña o cerca de las ternereras los "sitios de limpieza" utilizados, donde este último lugar representaría un riesgo para la bioseguridad de los terneros.

En general, podemos mencionar que existiría una gran falencia en la implementación de condiciones sanitarias básicas para los trabajadores, ya que muy pocas lecherías contaban con baños adecuados, comedores, vestidores, e incluso algunos predios mencionaron no tener agua potable para sus funcionarios.

\section{Maquinaria y equipos}

De acuerdo a la encuesta, la mayoría de las lecherías (30 de ellas; 90,9\%) utilizaba un equipo distinto para manejar el alimento y el estiércol de los bovinos, donde el uso del carro forrajero para la entrega de la alimentación (en aquellas lecherías que lo poseen) limitaría enormemente esta práctica

\section{CONCLUSIONES}

En general la situación de bioseguridad en lecherías encuestadas de la Zona Central de Chile es insuficiente. El área con el menor número de medidas de bioseguridad implementadas, correspondió al sistema de control e ingreso de personas externas al predio, donde en la mayoría de las lecherías no existían restricciones para las visitas. Otro punto importante a destacar, es que en todas las lecherías evaluadas se constató la existencia de otras especies de animales domésticos. En la mayoría de los casos, estas especies poseían contacto directo con los bovinos o con sus fuentes de agua o de alimento.

Sin embargo, la incorporación de animales al plantel desde otros predios es una práctica poco utilizada en las lecherías encuestadas, ya que la mayoría de los productores reconoce que mantener un rebaño cerrado es una medida fundamental para prevenir el ingreso de patógenos al plantel.

Se considera además necesario educar y capacitar al personal sobre aspectos de bioseguridad predial, en especial sobre el uso de material desechable, manejo de animales enfermos, existencia de otros animales, manejo del ternero recién nacido y técnicas de limpieza e higiene.

Por último, con los resultados finales de la encuesta y tomando en consideración la opinión de los colaboradores y bibliografía consultada, se debería continuar con la implementación 
de un plan básico de bioseguridad predial orientado a planteles lecheros de la Zona Central de Chile y la capacitación del personal involucrado.

\section{Agradecimientos}

Este estudio fue financiado por el Fondo ce Investigaciones Veterinarias (FIV) de la Facultad de Ciencias Veterinarias y Pecuarias de la Universidad de Chile por apoyarnos en la realización este estudio. Se agradece a los jefes de lechería, administradores y veterinarios que participaron y colaboraron en las encuestas e inspecciones de los predios evaluados, y al Dr. Luis Moraga por la colaboración en este proyecto. 


\section{REFERENCIAS}

BAREFOOT, S. 2002. Dairy farm biosecurity and food safety. Workshop on Terrorism and Biosecurity. Cayce, South Carolina, USA.

BASSO, W.; VENTURINI, L.; VENTURINI, M. 2001. First isolation of Neospora caninum from the feces of a naturally infected dog. J. Parasitol., 87: 612-618.

DARGATZ, D.A.; GARRY, F.B.; TRAUB-DARGTZ, J.L. 2002. An introduction to biosecurity of cattle operations. Vet. Clin. North Am. Food Anim. Pract., 18:1-5.

DUBEY, J.; BARR, B.; BARTA, J. 2002. Redescription of Neospora caninum and its differentiation from related coccidia. Int. J. Parasitol., 32: 929-946.

HOE, F.; RUEGG, P. 2006. Opinions and practices of Wisconsin dairy producers about biosecurity and animal well-being. J. Dairy Sci., 89(6):2297-2308.

LINDSAY, D.; DUBEY, J.; DUNCAN, R. 1999. Confirmation that the dog is a definitive host for Neospora caninum. Vet. Parasitol., 82: 327-333.

MCALLISTER, M.; DUBEY, J.; LINDSAY, D., JOLLEY, W.; WILLS, R.; MCGUIRE, A. 1998. DogS are definitive hosts of Neospora caninum. Int. J. Parasitol., 28: 1473-1478.

RADOSTITS, O. 2001. Control of Infectious Diseases of Food-Producing Animals. En su: Herd Health. $3^{\circ}$ edición. United States of America. W.B. Saunders Company, pp. 147-188.

RADOSTITS, O. 2003 Principios de bioseguridad para la producción de carne y leche. Memorias del V Simposio Internacional de Reproducción Animal, Córdoba, Argentina. pp 322 $-367$.

STABEL, J.; HURD, S.; CALVENTE, L.; ROSENBUSCH, R. 2004. Destruction of Mycobacterium paratuberculosis, Salmonella spp., and Mycoplasma spp. In Raw Milk by a Commercial OnFarm High-Temperature, Short-Time Pasteurizer. J. Dairy Sci., 87: 2177-2183.

USDA, UNITED STATES DEPARTMENT OF AGRICULTURE. 2004. Animal Disease Exclusion Practices on U.S. Dairy Operations: 2002. USDA: APHIS: VS, CEAH, National Animal Health Monitoring System, United States Department of Agriculture, 48 p.

WELLS, S. 2000. Symposium: Biosecurity. Biosecurity on Dairy Operations: Hazards and Risks. J. Dairy Sci., 83(10): 2380-2386.

WELLS, S.J. 2002. Biosecurity for gastrointestinal diseases of adult dairy cattle. Vet. Clin. North Am. Food Anim. Pract., 18:35-55. 
WOLFGANG, D.R., 2002. Biosecurity - A practical approach [ en línea ] Penn State University, College of Agricultural Sciences, Cooperative Extension, Veterinary Science Information. 